\title{
The Emergence of Multiple Myeloma in a Patient with Essential Thrombo- cythemia: A Case Report
}

\author{
Terzi ${ }^{*}{ }^{1}$, Korkmaz $\mathrm{S}^{1}$, Kaya OB ${ }^{2}$, Kaçan $\mathrm{T}^{3}$ and Şencan $\mathrm{M}^{1}$
}

${ }^{1}$ Cumhuriyet University, Department of Hematology, Sivas, Turkey

${ }^{2}$ Cumhuriyet University, Department of Internal Medicine, Sivas, Turkey

${ }^{3}$ Cumhuriyet University, Department of Oncology, Sivas, Turkey

${ }^{*}$ Corresponding author: Terzi H, Cumhuriyet University, Medical Faculty, Department of Hematology, Turkey, E-mail: dr.terzi@hotmail.com

Citation: Terzi H, Korkmaz S, Kaya OB, Kaçan T, Şencan M (2015) The Emergence of Multiple Myeloma in a Patient with Essential Thrombocythemia: A Case Report. J Hematol Blood Disord 1(1): 101. doi: 10.15744/2455-7641.1.101

Received Date: January 16, 2015 Accepted Date: July 31, 2015 Published Date: August 03, 2015

\section{Abstract}

Multiple myeloma is a neoplastic disease characterized by neoplastic proliferation of plasma cells and monoclonal protein expression and related tissue damage. In the presence of overt clinical signs, the disease can easily be diagnosed, however in order to diagnose atypical cases, further investigation is needed with high clinical suspicion. Essential thrombocythemia is a chronic myeloproliferative neoplasm which leads to an increase in platelet count with circulating megakaryocytes. When compared with other myeloproliferative neoplasms the prognosis is better and is more common in women. Herein we report a 68-year old patient suffering of essential thombocythemia, who later in follow-up developed multiple myelomas.

Keywords: Multiple myeloma; Essential thrombocythemia

\section{Introduction}

Multiple myeloma (MM) effectuates about $1 \%$ of cancer cases and $10 \%$ of hematologic malignancies, while average of introductory age is 66 with an incidence of 3-4/100.000 [1]. The major clinical manifestation of MM is bone pain, especially in the back and the chest wall, while laboratory findings include hypercalcemia, high sedimentation rate, anemia (usually normocytic and normochromic), and lytic bone lesions. In $80 \%$ of patients with lytic bone lesions detected by bone scan, osteopenia, osteoporosis or pathologic fractures are identified. Kidney uptake rate is $48 \%$ in MM and light chains are usually monitored [2]. Essential thrombocythemia (ET) is a chronic myeloproliferative neoplasm (MPN) which leads to an increase in the number of platelets and manifests itself by continuous proliferation of megakaryocytes. ET might be seen as a secondary malignancy such as nonHodgkin's lymphoma (NHL) and myelodysplastic syndrome (MDS). However, the occurrence of ET with MM is very scarcely reported in the literature.

\section{Case Report}

A 68-year old male patient was admitted to our clinic with back pain, weight loss, and fatigue. Laboratory findings revealed anemia, thrombocytosis, and elevated level of erythrocyte sedimentation rate (ESR). Because of the clinical findings, serum immunofixation electrophoresis, bone marrow aspiration and biopsy were performed. Serum immunofixation electrophoresis revealed IgA lambda monoclonal gammopathy. Serum total and free kappa and lambda values were 114 and $693 \mathrm{mg} / \mathrm{dL}$, respectively. A plasma cell infiltration was $20 \%$ on his bone narrow aspiration and CD138-positive lambda light chain positive plasma cell infiltration was $20 \%$ on his bone marrow biopsy. As a result, the patient was diagnosed who developed IgA-lambda MM. 3 cycles of vincristine, adriamycin, dexamethasone (VAD) chemotherapy and zoledronic acid were administered. In follow-up, the clinical signs regressed and the control bone marrow aspiration and biopsy revealed 8-10\% plasma cell infiltration. Owing to continuation of monoclonal gammopathy in serum immunofixation, bortezomib plus cyclophosphamide and dexamethasone combination chemotherapy (VCD) was started. After 3 cycles of VCD chemotherapy, 1-2\% of plasma cells was detected by control bone marrow aspiration and biopsy. Polyclonal gammopathy was found in serum immunofixation assay. Autologous stem cell transplantation was performed. The patient is still in remission without any maintenance treatment, and he is being followed up regularly.

In his history, the patient admitted to hospital with complaining of headache, dizziness, tinnitus, and itching two years ago. On his laboratory findings, complete blood count revealed 16,000 white blood cells, $13 \mathrm{~g} / \mathrm{dL}$ hemoglobin, and 1000x10 3 platelets. Although the physical examination of the patients were normal, bone marrow aspiration and biopsy were performed due to exclude reactive thrombocytosis and MPN. Bone marrow aspiration and biopsy were hypercellular, and there was an increase in number of megakaryocytes. BCR-ABL was negative and JAK2 (V617F) mutation was present. The patient was diagnosed with ET, and hydroxyurea, acetylsalicylic acid and allopurinol were started. During the follow-up period the patient's clinical and laboratory findings was improved. In this case, we report a 68-year old male patient who developed IgA-lambda MM more than 2 years after the diagnosis of ET. 


\section{Discussion}

ET is a philadelphia chromosome negative chronic MPN which is characterized by an increase in the number of circulating platelets in the peripheral blood and an increased risk of thrombohemorrhagic complications due to excessive proliferation of megakaryocytes in the bone marrow [3]. ET has a risk of progression to myelofibrosis or acute myeloid leukemia. However, the most important complications include common thrombohemorrhagic events that significantly affect the life expectancy of patients [4]. Patients with ET can be detected by some complications such as thrombotic events, vertigo, tinnitus, light microcirculatory disorders, ischemic stroke, transient ischemic attack, myocardial infarction, cerebral sinus venous thrombosis or deep vein thrombosis in preclinical phase of the disease or after diagnosis.

The JAK2 V617F mutation is detected in approximately 50-60\% of patients with ET [5]. The relationship between JAK2 V617F mutation and the tendency of increased thrombosis have been already reported. However, increased peripheral platelet numbers in the pathogenesis of thrombotic events and the role of the main cardiovascular risk factors is still controversial in ET [5-7].

In ET, hydroxyurea is recommended as first-line treatment. On the other hand, drugs such as interferon alpha or non-leukaemogenic anagrelide are recommended to high risk patients who are resistant or intolerant to hydroxyurea treatment. Cytoreductive therapy is indicated for high-risk patients with developing thrombohemorrhagic events [8]. Secondary neoplasms in patients with ET during their follow-up period have been scarcely reported in the literature. There are some reports in the literature. Secondary hematologic malignancies more often includes NHL and MDS but the development of MM is extremely rare [9,10]. In studies performed previously in the development of secondary malignancies, during ET treatment having effects of used toxic drugs were drawn attention [11]. As a consequence, development of secondary malignancies is always possible in patients with ET. In this case, MM was identified as secondary malignancy. Furthermore, we claim that patients who were administered cytotoxic treatment should be carefully assessed against the risk of developing secondary malignancies at each visit.

\section{References}

1. Phekoo KJ, Schey SA, Richards MA, Bevan DH, Bell S, et al. (2004) A population study to define the incidence and survival of multiple myeloma in a National Health Service Region in UK. Br J Haematol 127: 299-304.

2. Kyle RA, Gertz MA, Witzig TE, Lust JA, Lacy MQ, et al. (2003) Review of 1027 patients with newly diagnosed multiple myeloma. Mayo Clinc proc 78: 21-33.

3. Tefferi A, Vardiman JW (2008) Classification and diagnosis of myeloproliferative neoplasms: the 2008 World Health Organization criteria and point-of-care diagnostic algorithms. Leukemia 22: 14-22.

4. Falanga A, Marchetti M (2012) Thrombotic disease in the myeloproliferative neoplasms. Hematology Am Sco Hematol Educ program 2012: 571-81.

5. Bench AJ, White HE, Foroni L, Godfrey AL, Gerrard G, et al. (2013) Molecular diagnosis of the myeloproliferative neoplasms: UK guidelines for the detection of JAK2 V617F and other relevant mutations. Br J Haematol 160: 25-34.

6. Tefferi A, Elliott M (2007) Thrombosis in myeloproliferative disorders: prevalence, prognostic factors, and the role of leukocytes and JAK2V617F. Semin Thromb Hemost 33: 313-20.

7. Carobbio A, Thiele J, Passamonti F, Rumi E, Ruggeri M, et al. (2011) Risk factors for arterial and venous thrombosis in WHO-defined essential thrombocythemia: an international study of 891 patients. Blood 117: 5857-9.

8. Barbui T, Barosi G, Birgegard G, Cervantes F, Finazzi G, et al. (2011) Philadelphia-negative classical myeloproliferative neoplasms: critical concepts and management recommendations from European LeukemiaNet. J Clin Oncol 29: 761-70.

9. Bjorkholm M, Derolf AR, Hultcrantz M, Kristinsson SY, Ekstrand C, et al. (2011) Treatment-related risk factors for transformation to acute myeloid leukemia and myelodysplastic syndromes in myeloproliferative neoplasms. J Cli Oncol 29: 2410-5.

10. Radaelli F, Onida F, Rossi FG, Zilioli VR, Colombi M, et al. (2008) Second malignancies in essential thrombocythemia (ET): a retrospective analysis of 331 patients with long-term follow-up from a single institution. Hematology 13: 195-202.

11. Eskazan AE, Ongoren S, Ar MC, Soysal T, Ferhanoglu B, et al. (2011) Essential thrombocythemia and multiple myeloma: two rare diseases in one patient. Clin Lymphoma Myeloma Leuk 11: 442-5.

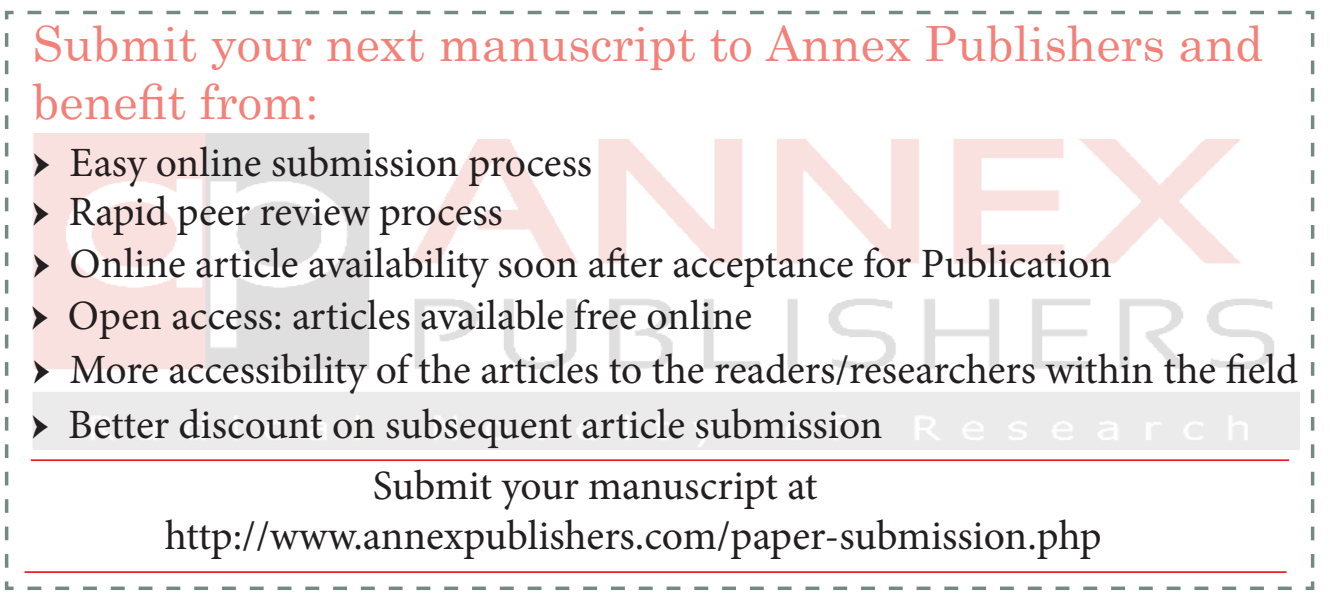

